MATHEMATICS OF COMPUTATION

Volume 75, Number 253, Pages 475-484

S 0025-5718(05)01777-1

Article electronically published on September 9, 2005

\title{
AN OLD CONJECTURE OF ERDÖS-TURÁN ON ADDITIVE BASES
}

\author{
PETER BORWEIN, STEPHEN CHOI, AND FRANK CHU
}

Abstract. There is a 1941 conjecture of Erdős and Turán on what is now called additive basis that we restate:

Conjecture 0.1 (Erdős and Turán). Suppose that $0=\delta_{0}<\delta_{1}<\delta_{2}<\delta_{3} \ldots$ is an increasing sequence of integers and

$$
s(z):=\sum_{i=0}^{\infty} z^{\delta_{i}} .
$$

Suppose that

$$
s^{2}(z):=\sum_{i=0}^{\infty} b_{i} z^{i} .
$$

If $b_{i}>0$ for all $i$, then $\left\{b_{n}\right\}$ is unbounded.

Our main purpose is to show that the sequence $\left\{b_{n}\right\}$ cannot be bounded by 7 . There is a surprisingly simple, though computationally very intensive, algorithm that establishes this.

\section{INTRODUCTION}

Suppose that $0=\delta_{0}<\delta_{1}<\delta_{2}<\delta_{3} \cdots$ is an increasing sequence of integers and

$$
s(z)=\sum_{i=0}^{\infty} z^{\delta_{i}}
$$

and

$$
s^{2}(z):=\sum_{i=0}^{\infty} b_{i} z^{i}
$$

with $b_{i}>0$ for all $i$. Then the set $A:=\left\{\delta_{1}<\delta_{2}<\delta_{3} \cdots\right\}$ is called a basis of order two, or simply a basis (that is, every natural number can be written as a sum of two elements of $A$ ). Hence the Erdős and Turán conjecture can be rephrased: for any basis, the number of representations is unbounded.

We prove, as our main result, the following theorem.

Theorem 1.1. Suppose that each $a_{i}$ is a nonnegative integer, and

$$
f(z):=\sum_{i=0}^{\infty} a_{i} z^{i} .
$$

Received by the editor September 28, 2004 and, in revised form, November 15, 2004.

2000 Mathematics Subject Classification. Primary 11B83, 05B20; Secondary 94A11, 68R05.

Key words and phrases. Erdős and Turán conjecture, additive basis.

This research was supported in part by grants from NSERC of Canada and MITACS.

The third author was supported by the NSERC Undergraduate Student Research Award. 
Suppose that

$$
f^{2}(z):=\sum_{i=0}^{\infty} b_{i} z^{i}
$$

If $b_{i}>0$ for all $i$, then $\sup \left\{b_{i}\right\} \geq 8$. In other words, the maximum number of representations of any basis is $\geq 8$.

This improves a previous result by Grekos, Haddad, Helou and Pihko in [5], where they prove that the maximum number of representations of any basis is $\geq 6$. The method we employ is surprisingly simple, though computationally very intensive. The methods in [5] involve considerably more analysis and less computation.

Erdős and Turán 3 raised this problem in a 1941 paper on Sidon sets and related problems and not in the language of additive basis.

On page 48 of "Old and New problems and results in combinatorial number theory", by Erdös and Graham [4, the conjecture is one of the open problems, and there is a $\$ 500$ prize offered. (The problem is described in terms of bases of order 2 after a discussion of bases.) Erdős and Graham 4 also ask for an explicit construction where

$$
1 \leq b_{i}=o\left(i^{\epsilon}\right)
$$

for all positive $\epsilon$.

If the sequence of $\delta_{i}$ is allowed to include negative integers, then the conjecture is false. This is due to Nathanson [6. In this case there is a simple explicit construction. For other related problems, we refer to [1], 2] and [7.

\section{ERDŐS-TURÁN CONJECTURE}

This old conjecture restates in a number of ways.

Conjecture 2.1 (Erdős and Turán-original version). Suppose that each $a_{i}$ is a nonnegative integer, and

$$
f(z):=\sum_{i=0}^{\infty} a_{i} z^{i}
$$

Suppose that

$$
f^{2}(z):=\sum_{i=0}^{\infty} b_{i} z^{i}
$$

If $b_{i}>0$ for all but finitely many $i$, then $\left\{b_{n}\right\}$ is unbounded.

This is in fact equivalent to the (apparently stronger) statement below. This follows from noticing that adding any finite number of positive terms to the sum $f(z)$ does not change the boundedness of $\left\{b_{n}\right\}$.

Conjecture 2.2 (Erdős and Turán-version 2). Suppose that each $a_{i}$ is a nonnegative integer, and

$$
f(z):=\sum_{i=0}^{\infty} a_{i} z^{i}
$$

Suppose that

$$
f^{2}(z):=\sum_{i=0}^{\infty} b_{i} z^{i}
$$

If $b_{i}>0$ for all $i$, then $\left\{b_{n}\right\}$ is unbounded. 
In this conjecture, in consideration of what happens if each positive integer $a_{i}$ in a minimal example is replaced by 1 , it is obviously necessary and sufficient to consider series of the form

$$
s(z):=\sum_{i=0}^{\infty} z^{\delta_{i}},
$$

where $0=\delta_{0}<\delta_{1}<\delta_{2}<\delta_{3} \cdots$ and each $\delta_{i}$ is an integer. This is the form of the conjecture we analyze.

Conjecture 2.3 (Erdős and Turán-version 3). Suppose that $0=\delta_{0}<\delta_{1}<\delta_{2}<$ $\delta_{3} \cdots$ is an increasing sequence of integers, and

$$
s(z)=\sum_{i=0}^{\infty} z^{\delta_{i}} \text {. }
$$

Suppose that

$$
s^{2}(z):=\sum_{i=0}^{\infty} b_{i} z^{i} .
$$

If $b_{i}>0$ for all $i$, then $\left\{b_{n}\right\}$ is unbounded.

From now on we suppose that $0=\delta_{0}<\delta_{1}<\delta_{2}<\delta_{3} \cdots$ is an increasing sequence of integers.

Lemma 2.4. For $n \geq 0$ let

$$
s_{n}(z):=\sum_{i=0}^{n} z^{\delta_{i}}
$$

and let (the finite sum)

$$
s_{n}^{2}(z):=\sum_{i=0}^{\infty} B_{i}(n) z^{i} .
$$

Then we have $B_{i}(n) \leq B_{i}(n+1)$ for all $i \geq 0$ and $B_{i}(n)=B_{i}(n+1)$ for $i=$ $0,1, \ldots, \delta_{n}$.

Proof. It follows easily from

$$
B_{i}(n)=\#\left\{\delta_{j}+\delta_{l}=i: 0 \leq j, l \leq n\right\},
$$

and $\left\{\delta_{n}\right\}$ is a strictly increasing sequence.

Corollary 2.5. Suppose that

$$
s(z)=\sum_{i=0}^{\infty} z^{\delta_{i}}
$$

and suppose that

$$
s^{2}(z)=\sum_{i=0}^{\infty} b_{i} z^{i}
$$

has each

$$
b_{i}>0
$$

Then

$$
s_{n}(z)=\sum_{i=0}^{n} z^{\delta_{i}}
$$


and

satisfy

$$
s_{n}^{2}(z)=\sum_{i=0}^{\infty} B_{i} z^{i}
$$

and

$$
B_{i}>0, i=0,1, \ldots, \delta_{n},
$$

$$
\max \left\{B_{i}\right\} \leq \max \left\{b_{i}\right\} .
$$

Proof. In view of Lemma 2.4, $B_{i} \leq b_{i}$ for all $i \geq 0$ and $B_{i}=b_{i}$ for $i=0,1, \ldots, \delta_{n}$. The corollary follows.

Lemma 2.6. Fix $k$ and let $E_{n}(k)$ denote the set of polynomials of the form

$$
s_{n}(z)=\sum_{i=0}^{n} z^{\delta_{i}}
$$

where $0=\delta_{0}<\delta_{1}<\delta_{2}<\cdots<\delta_{n}$ and where

$$
s_{n}^{2}(z)=\sum_{i=0}^{\infty} B_{i} z^{i}
$$

with

$$
B_{i}>0, i=0,1, \ldots, \delta_{n},
$$

and

$$
\max \left\{B_{i}\right\} \leq k .
$$

Then each element of $p(z) \in E_{n}(k)$ is an extension of an element of $q(z) \in E_{n-1}(k)$ of at most one more than twice the degree, in the sense that

$$
p(z):=z^{\gamma}+q(z),
$$

where

$$
\text { degree }(q)<\gamma \leq 2 \text { degree }(q)+1 .
$$

In particular, the largest degree of $p(z)$ in $E_{n}(k)$ is at most $n^{2}+2 n-2$ and

$$
\left|E_{n}(k)\right| \leq\left(n^{2}-2\right)\left|E_{n-1}(k)\right| .
$$

Proof. If $s_{n}(z)=1+z^{\delta_{1}}+\cdots+z^{\delta_{n}} \in E_{n}(k)$ with $0=\delta_{0}<\delta_{1}<\cdots<\delta_{n}$, then from Lemma 2.4, we have $B_{i}(n-1) \leq B_{i}(n) \leq k$ for all $i$ and $B_{i}(n-1)=B_{i}(n)>0$ for $i=0,1, \ldots, \delta_{n-1}$. Hence $s_{n-1}(z)$ belongs to $E_{n-1}(k)$ and $s_{n}(z)=z^{\delta_{n}}+s_{n-1}(z)$. Furthermore, if $\delta_{n}>2 \delta_{n-1}+1$, then $\delta_{i}+\delta_{j} \neq 2 \delta_{n-1}+1$ for $0 \leq i, j \leq n$ and hence

$$
B_{2 \delta_{n-1}+1}(n)=\#\left\{\delta_{i}+\delta_{j}=2 \delta_{n-1}+1: 0 \leq i, j \leq n\right\}=0 .
$$

This contradicts $s_{n}(z) \in E_{n}(k)$. Thus, we have

$$
\delta_{n-1}<\delta_{n} \leq 2 \delta_{n-1}+1 .
$$

On the other hand, we have

$$
\begin{aligned}
(n+1)^{2}=s_{n}(1)^{2} & =\sum_{i=0}^{2 \delta_{n}} B_{i} \\
& \geq\left(B_{0}+\cdots+B_{\delta_{n}}\right)+B_{\delta_{n}+1}+B_{2 \delta_{n}} .
\end{aligned}
$$

If $s_{n}(z) \in E_{n}(k)$, then $B_{0}, \ldots, B_{\delta_{n}} \geq 1$ and clearly $B_{2 \delta_{n}}=1$ and $B_{\delta_{n}+1} \geq 1$ because $\delta_{1}$ always equals 1 . Thus for $n \geq 1$, we have

$$
\delta_{n} \leq(n+1)^{2}-3=n^{2}+2 n-2 .
$$


Now since $s_{n}(z)=z^{\delta_{n}}+s_{n-1}(z)$, we have

$$
\left|E_{n}(k)\right| \leq\left(n^{2}-2\right)\left|E_{n-1}(k)\right|
$$

by (2.2) and (2.3).

From (2.1), if there is some $n_{0}$ such that $E_{n_{0}}(k)=\emptyset$, then $E_{n}(k)=\emptyset$ for all $n \geq n_{0}$.

The utility is that it suggests an algorithm for generating

$$
E(k):=E_{0}(k) \cup E_{1}(k) \cup E_{2}(k) \cup E_{3}(k) \ldots
$$

that terminates exactly when $E_{n}(k)$ is empty for some $n$. Hence we have the following result.

Theorem 2.7. In the notation of the previous corollary, if, for some $n, E_{n}(k)$ is empty or equivalently, $E(k)$ is finite, then no series of the form

$$
s(z):=\sum_{i=0}^{\infty} z^{\delta_{i}}
$$

where

$$
s^{2}(z):=\sum_{i=0}^{\infty} b_{i} z^{i},
$$

has each

$$
b_{i}>0
$$

and

$$
\max \left\{b_{i}\right\} \leq k
$$

can exist. Furthermore, if for every $k, E(k)$ is finite, then the Erdös and Turán conjecture is true.

Proof. Since $E(k)$ is finite if and only if, for some $n, E_{n}(k)$ is empty, so the theorem follows from Lemma 2.6

\section{Computations}

Each element of $p \in E_{n}(k)$ is an extension of an element of $q \in E_{n-1}(k)$ of at most twice the degree plus one. So $E_{n}(k)$ can be generated readily from $E_{n-1}(k)$ in at most $N M$ steps, where $N$ is the size of $E_{n-1}(k)$ and $M$ is one more than the largest degree of an element of $E_{n-1}(k)$.

So for example $E(3)$ is

$$
\begin{gathered}
\left\{1, x+1, x^{2}+x+1, x^{3}+x+1, x^{4}+x^{2}+x+1, x^{5}+x^{2}+x+1, x^{5}+x^{3}+x+1,\right. \\
\left.x^{7}+x^{4}+x^{2}+x+1, x^{8}+x^{5}+x^{2}+x+1\right\} .
\end{gathered}
$$

Here

$$
\begin{gathered}
E_{0}(3)=\{1\}, \\
E_{1}(3)=\{x+1\}, \\
E_{2}(3)=\left\{x^{2}+x+1, x^{3}+x+1\right\}, \\
E_{3}(3)=\left\{x^{4}+x^{2}+x+1, x^{5}+x^{2}+x+1, x^{5}+x^{3}+x+1\right\},
\end{gathered}
$$

and

$$
E_{4}(3)=\left\{x^{7}+x^{4}+x^{2}+x+1, x^{8}+x^{5}+x^{2}+x+1\right\} .
$$

Note how the elements extend. Also $E_{n}(3)=\emptyset$ for all $n>4$. 
It is a computation that $E(4)$ contains exactly 404 elements all of degree 40 or less, and $E(5)$ contains exactly 6355 elements all of degree 52 or less. A typical element is

$$
t:=x^{41}+x^{36}+x^{30}+x^{27}+x^{21}+x^{15}+x^{13}+x^{8}+x^{5}+x^{4}+x^{3}+x+1
$$

with

$$
\begin{aligned}
t^{2}:= & x^{82}+2 x^{77}+x^{72}+2 x^{71}+2 x^{68}+2 x^{66}+2 x^{63}+2 x^{62}+x^{60}+4 x^{57}+2 x^{56} \\
& +3 x^{54}+4 x^{51}+4 x^{49}+2 x^{48}+2 x^{46}+4 x^{45}+4 x^{44}+2 x^{43}+5 x^{42}+4 x^{41} \\
& +4 x^{40}+2 x^{39}+2 x^{38}+2 x^{37}+4 x^{36}+4 x^{35}+4 x^{34}+2 x^{33}+2 x^{32}+4 x^{31} \\
& +5 x^{30}+2 x^{29}+4 x^{28}+2 x^{27}+3 x^{26}+2 x^{25}+2 x^{24}+2 x^{23}+2 x^{22}+4 x^{21} \\
& +2 x^{20}+2 x^{19}+4 x^{18}+2 x^{17}+5 x^{16}+2 x^{15}+2 x^{14}+4 x^{13}+2 x^{12}+2 x^{11} \\
& +x^{10}+4 x^{9}+5 x^{8}+2 x^{7}+3 x^{6}+4 x^{5}+4 x^{4}+2 x^{3}+x^{2}+2 x+1 .
\end{aligned}
$$

A first attempt at computing $E(6)$ shows very quickly that it is much larger than that of $E(5)$. So the straightforward breadth first search has to be optimized in several ways to compute $E(6)$ and $E(7)$.

First, the search needs to be depth first, because the intermediate sets $E_{n}(6)$ are large. (A trial execution of the breadth-first algorithm showed us that $E_{17}(6)$ has as many as 200 million sets! This is clearly a memory-wise problem.)

Another key optimization is to prune the search during an extension, as the following lemma allows.

Lemma 3.1. Let $s_{n}(z) \in E_{n}(k)$ so

$$
s_{n}(z)=\sum_{i=0}^{n} z^{\delta_{i}},
$$

where $0=\delta_{0}<\delta_{1}<\delta_{2}<\cdots<\delta_{n}$ and where

$$
s_{n}^{2}(z)=\sum_{i=0}^{\infty} B_{i} z^{i}
$$

with

$$
B_{i}>0, i=0,1, \ldots, \delta_{n}
$$

and

$$
\max \left\{B_{i}\right\} \leq k .
$$

Suppose $\phi$ is the first index such that $B_{\phi}=0$. Then

$$
\delta_{n}<\phi \leq 2 \delta_{n}+1 \text {, }
$$

and any extension $s_{n+1}(z) \in E_{n+1}(k)$ of $s_{n}(z)$ is of the form

$$
s_{n+1}(z)=s_{n}(z)+z^{\gamma},
$$

where

$$
\gamma \leq \phi
$$

Proof. The proof is similar to Lemma 2.4. 
TABLE 1. Results of computation

\begin{tabular}{|c|c|c|c|}
\hline$k$ & $|E(k)|$ & maxlength $(k)$ & maxdegree $(k)$ \\
\hline \hline 2 & 3 & 3 & 3 \\
\hline 3 & 9 & 5 & 8 \\
\hline 4 & 404 & 12 & 40 \\
\hline 5 & 6,355 & 14 & 52 \\
\hline 6 & $11,482,910,373$ & 35 & 264 \\
\hline 7 & $1,268,361,281,038$ & 41 & 328 \\
\hline
\end{tabular}

These optimizations are only heuristics and do not better the time complexity of the algorithm. However, they prove to be valuable in speeding up the searches and make the computation of $E(6)$ and $E(7)$ feasible.

We give the size of $E(k)$ in Table 1, and the complete results in Table 3 . To present our results, we define

$$
\operatorname{maxlength}(k)=\sup \left\{n+1: s_{n} \in E(k)\right\}
$$

and

$$
\operatorname{maxdegree}(k)=\sup \left\{\operatorname{deg}\left(s_{n}\right): s_{n} \in E(k)\right\} .
$$

$E(6)$ was computed in approximately 6.5 hours on a personal computer with clock speed about $2.2 \mathrm{GHz}$. The size of $E(7)$ was evidently too large for a single computer to handle. To aid the computation, we used XGrid, Apple's distributed system.

XGrid is a software that lets us turn our cluster of Apple G4's into a parallel distributed system. It provides parallel computation by queuing multiple jobs and distributing them to the cluster when there are free resources. Our algorithm lends itself nicely to distributed computing, as searches starting on different elements of the same size are completely independent.

So, to distribute $E(7)$, we first compute $E_{6}(7)$, which has 65 elements. We then submit a job to XGrid for each of these 65 elements (we had 65 G4's at our disposal), using it as a starting point of the search. We then combine the results of each of these jobs.

The current design of XGrid will run each job independently, on an individual computer at a given time. Thus, the power we harnessed from XGrid was equivalent to 65 G4's running individually. The total time it took XGrid to finish its computations was about one month.

We see, from the results, a dramatic increase in size between $E(5)$ (around 7 thousand) and $E(6)$ (around 11 billion). If the growth rate maintains, then the size of $E(7)$ should have been at least a million times larger than that of $E(6)$. However, that is not the case.

This behavior is tied to the parity of $k$. We believe that when going from an even value of $k$ to an odd value of $k$, the increase in size will not be as dramatic as going from an odd value to an even value. The argument is that the $B_{i}$ 's in $s_{n}^{2}(z)$ will increase by 2 when some distinct pair $z^{j}, z^{k}$ is in $s_{n}(z)$ where $j+k=i$, and only by 1 if the pair is not distinct. The latter case is much less frequent than the first. As such, when $k$ is small (say $<15$ ), the parity of $k$ is likely to have a big effect on the size of $E(k)$. 
TABLE 2. Lower bound on maxlength for $6 \leq k \leq 11$

\begin{tabular}{|c|c|}
\hline$k$ & min maxlength $(k)$ \\
\hline \hline 6 & 35 \\
\hline 7 & 39 \\
\hline 8 & 70 \\
\hline 9 & 76 \\
\hline 10 & 127 \\
\hline 11 & 134 \\
\hline
\end{tabular}

It is likely that this parity argument will break down when $k$ is large. However, as for our case, it is a legitimate conjecture, as reflected in the size of $E(7)$. The size of $E(7)$ is only about 100 times bigger than $E(6)$, and maxlength(7) is also not much greater than maxlength(6). We have even stronger support for this argument in our preliminary results for $E(8)$ and up in Table 2.

The results in Table 2 were obtained by running our algorithm for one hour and collecting the largest polynomial we discover. As can be seen, there is a much bigger difference when going from an odd $k$ to an even $k$, than from an even $k$ to an odd $k$. Although these results give only the lower bound, the pattern it exhibits is far from being coincidental. At the very least, $|E(8)|$ is quite a few magnitudes larger than $|E(7)|$, and we believe our current algorithm will not be able to compute $E(k)$ for $k \geq 8$ in any reasonable amount of time. It seems reasonable to believe that the size of $E(k)$ grows exponentially, or even faster, with respect to $k$.

Table 3 shows data for the size of $E_{n}(k)$. Note that for each value of $k$, the $E_{n}(k)$ values follow an interesting pattern. The number of digits seem to grow linearly with respect to $n$, until in the middle, when it starts to fall linearly again.

TABle 3. Complete results

\begin{tabular}{|c|c|c|c|c|c|c|}
\hline$k$ & \multicolumn{2}{|l|}{3} & \multicolumn{2}{|l|}{4} & \multicolumn{2}{|l|}{5} \\
\hline$|E(k)|$ & 9 & & 404 & & 6,355 & \\
\hline maxlength $(k)$ & 5 & & 12 & & 14 & \\
\hline maxdegree $(k)$ & 8 & & 40 & & 52 & \\
\hline & $\begin{array}{l}\left|E_{1}(3)\right| \\
\left|E_{2}(3)\right| \\
\left|E_{3}(3)\right| \\
\left|E_{4}(3)\right| \\
\left|E_{5}(3)\right|\end{array}$ & $\begin{array}{l}=1 \\
=1 \\
=2 \\
=3 \\
=2\end{array}$ & $\begin{array}{l}\left|E_{1}(4)\right| \\
\left|E_{2}(4)\right| \\
\left|E_{3}(4)\right| \\
\left|E_{4}(4)\right| \\
\left|E_{5}(4)\right| \\
\mid E_{6}(4) \\
\left|E_{7}(4)\right| \\
\left|E_{8}(4)\right| \\
\left|E_{9}(4)\right| \\
\left|E_{10}(4)\right| \\
\left|E_{11}(4)\right| \\
\left|E_{12}(4)\right|\end{array}$ & $\begin{array}{l}=1 \\
=1 \\
=2 \\
=5 \\
=15 \\
=38 \\
=89 \\
=122 \\
=86 \\
=38 \\
=6 \\
=1\end{array}$ & $\begin{array}{c}\left|E_{1}(5)\right| \\
\left|E_{2}(5)\right| \\
\left|E_{3}(5)\right| \\
\left|E_{4}(5)\right| \\
\left|E_{5}(5)\right| \\
\left|E_{6}(5)\right| \\
\left|E_{7}(5)\right| \\
\left|E_{8}(5)\right| \\
\left|E_{9}(5)\right| \\
\left|E_{10}(5)\right| \\
\left|E_{11}(5)\right| \\
\left|E_{12}(5)\right| \\
\left|E_{13}(5)\right| \\
\left|E_{14}(5)\right|\end{array}$ & $\begin{array}{l}=1 \\
=1 \\
=2 \\
=5 \\
=17 \\
=60 \\
=201 \\
=552 \\
=1,100 \\
=1,568 \\
=1,580 \\
=937 \\
=285 \\
=46\end{array}$ \\
\hline
\end{tabular}


Table 3. (continued)

\begin{tabular}{|c|c|c|c|c|}
\hline$k$ & \multicolumn{2}{|l|}{6} & \multicolumn{2}{|l|}{7} \\
\hline$|E(k)|$ & \multicolumn{2}{|c|}{$11,482,910,373$} & \multicolumn{2}{|c|}{$1,268,361,281,038$} \\
\hline $\operatorname{maxlength}(k)$ & \multicolumn{2}{|c|}{35} & \multicolumn{2}{|c|}{41} \\
\hline $\operatorname{maxdegree}(k)$ & \multicolumn{2}{|l|}{264} & \multicolumn{2}{|l|}{328} \\
\hline & $\begin{array}{l}\left|E_{1}(6)\right| \\
\left|E_{2}(6)\right| \\
\left|E_{3}(6)\right| \\
\left|E_{4}(6)\right| \\
\left|E_{5}(6)\right| \\
\left|E_{6}(6)\right| \\
\left|E_{7}(6)\right| \\
\left|E_{8}(6)\right| \\
\left|E_{9}(6)\right| \\
\left|E_{10}(6)\right| \\
\left|E_{11}(6)\right| \\
\left|E_{12}(6)\right| \\
\left|E_{13}(6)\right| \\
\left|E_{14}(6)\right| \\
\left|E_{15}(6)\right| \\
\left|E_{16}(6)\right| \\
\left|E_{17}(6)\right| \\
\left|E_{18}(6)\right| \\
\left|E_{19}(6)\right| \\
\left|E_{20}(6)\right| \\
\left|E_{21}(6)\right| \\
\left|E_{22}(6)\right| \\
\left|E_{23}(6)\right| \\
\left|E_{24}(6)\right| \\
\left|E_{25}(6)\right| \\
\left|E_{26}(6)\right| \\
\left|E_{27}(6)\right| \\
\left|E_{28}(6)\right| \\
\left|E_{29}(6)\right| \\
\left|E_{30}(6)\right| \\
\left|E_{31}(6)\right| \\
\left|E_{32}(6)\right| \\
\left|E_{33}(6)\right| \\
\left|E_{34}(6)\right| \\
\left|E_{35}(6)\right|\end{array}$ & $\begin{array}{l}=1 \\
=1 \\
=2 \\
=5 \\
=17 \\
=65 \\
=287 \\
=1,321 \\
=6,343 \\
=30,221 \\
=139,151 \\
=603,811 \\
=2,426,694 \\
=8,860,674 \\
=28,978,826 \\
=83,731,261 \\
=211,235,073 \\
=460,185,450 \\
=857,598,737 \\
=1,354,122,593 \\
=1,797,582,753 \\
=1,989,846,915 \\
=1,821,587,616 \\
=1,369,557,963 \\
=839,984,280 \\
=417,713,111 \\
=167,597,147 \\
=53,944,794 \\
=13,841,595 \\
=2,817,369 \\
=453,040 \\
=57,203 \\
=5,615 \\
=412 \\
=27\end{array}$ & \begin{tabular}{|l}
$\left|E_{1}(7)\right|$ \\
$\left|E_{2}(7)\right|$ \\
$\left|E_{3}(7)\right|$ \\
$\left|E_{4}(7)\right|$ \\
$\left|E_{5}(7)\right|$ \\
$\left|E_{6}(7)\right|$ \\
$\left|E_{7}(7)\right|$ \\
$\left|E_{8}(7)\right|$ \\
$\left|E_{9}(7)\right|$ \\
$\left|E_{10}(7)\right|$ \\
$\left|E_{11}(7)\right|$ \\
$\left|E_{12}(7)\right|$ \\
$\left|E_{13}(7)\right|$ \\
$\left|E_{14}(7)\right|$ \\
$\left|E_{15}(7)\right|$ \\
$\left|E_{16}(7)\right|$ \\
$\left|E_{17}(7)\right|$ \\
$\left|E_{18}(7)\right|$ \\
$\left|E_{19}(7)\right|$ \\
$\left|E_{20}(7)\right|$ \\
$\left|E_{21}(7)\right|$ \\
$\left|E_{22}(7)\right|$ \\
$\left|E_{23}(7)\right|$ \\
$\left|E_{24}(7)\right|$ \\
$\left|E_{25}(7)\right|$ \\
$\left|E_{26}(7)\right|$ \\
$\left|E_{27}(7)\right|$ \\
$\left|E_{28}(7)\right|$ \\
$\left|E_{29}(7)\right|$ \\
$\left|E_{30}(7)\right|$ \\
$\left|E_{31}(7)\right|$ \\
$\left|E_{32}(7)\right|$ \\
$\left|E_{33}(7)\right|$ \\
$\left|E_{34}(7)\right|$ \\
$\left|E_{35}(7)\right|$ \\
$\left|E_{36}(7)\right|$ \\
$\left|E_{37}(7)\right|$ \\
$\left|E_{38}(7)\right|$ \\
$\left|E_{39}(7)\right|$ \\
$\left|E_{40}(7)\right|$ \\
$\left|E_{41}(7)\right|$
\end{tabular} & $\begin{array}{l}=1 \\
=1 \\
=2 \\
=5 \\
=17 \\
=65 \\
=292 \\
=1,417 \\
=7,378 \\
=39,477 \\
=210,874 \\
=1,094,795 \\
=5,399,767 \\
=24,895,176 \\
=105,687,436 \\
=407,526,539 \\
=1,411,405,293 \\
=4,344,872,108 \\
=11,776,406,154 \\
=27,875,217,790 \\
=57,185,490,034 \\
=100,976,600,458 \\
=152,386,266,107 \\
=195,293,555,650 \\
=211,272,996,767 \\
=191,754,175,058 \\
=145,199,909,103 \\
=91,265,176,047 \\
=47,338,748,249 \\
=20,167,141,016 \\
=7,024,029,669 \\
=1,989,639,547 \\
=456,172,652 \\
=84,420,512 \\
=12,546,383 \\
=1,495,308 \\
=142,467 \\
=10,732 \\
=677 \\
=14 \\
=1\end{array}$ \\
\hline
\end{tabular}

\section{ACKNOWLEDGMENTS}

The authors would like to thank Ron Ferguson for his help in the computation of $E(7)$. 


\section{REFERENCES}

1. Martin Dowd, Questions related to the Erdös-Turán conjecture, SIAM J. Discrete Math. 1 (1988), no. 1, 142-150. MR0936616 (89h:11006)

2. P. Erdős and R. Frued, On Sidon-sequences and related problems, Mat. Lapok (New Ser.) (1991/2 (in Hungarian)), no. 1, 1-44.

3. P. Erdős and P. Turán, On a problem of Sidon in additive number theory, and on some related problems, J. London Math. Soc. 16 (1941), 212-215. MR0006197(3:270e)

4. P. Erdős and R. L. Graham, Old and new problems and results in combinatorial number theory: van der Waerden's theorem and related topics, Enseign. Math. (2) 25 (1979), no. 3-4, 325-344 (1980). MR0570317 (81f:10005)

5. G. Grekos, L. Haddad, C. Helou, and J. Pihko, On the Erdös-Turán conjecture, J. Number Theory 102 (2003), no. 2, 339-352. MR1997795 (2004j:11011)

6. Melvyn B. Nathanson, Unique representation bases for the integers, Acta Arith. 108 (2003), no. 1, 1-8. MR.1971077(2004c:11013)

7. Csaba Sándor, Range of bounded additive representation functions, Period. Math. Hungar. 42 (2001), no. 1-2, 169-177. MR1832703 (2002f:11010)

Department of Mathematics, Simon Fraser University, Burnaby, British Columbia, CANADA V5A 1S6

E-mail address: pborwein@cecm.sfu.ca

Department of Mathematics, Simon Fraser University, Burnaby, British Columbia, CANADA V5A 1S6

E-mail address: kkchoi@cecm.sfu.ca

Department of Mathematics, Simon Fraser University, Burnaby, British Columbia, CANADA V5A 1S6

E-mail address: pmc@cecm.sfu.ca 